\title{
Fitting a Graph to One-Dimensional Data*
}

\author{
Siu-Wing Cheng ${ }^{\dagger} \quad$ Otfried Cheong ${ }^{\ddagger} \quad$ Taegyoung Lee $^{\dagger} \quad$ Zhengtong Ren $^{\dagger}$
}

October 1, 2020

\begin{abstract}
Given $n$ data points in $\mathbb{R}^{d}$, an appropriate edge-weighted graph connecting the data points finds application in solving clustering, classification, and regresssion problems. The graph proposed by Daitch, Kelner and Spielman (ICML 2009) can be computed by quadratic programming and hence in polynomial time. While a more efficient algorithm would be preferable, replacing quadratic programming is challenging even for the special case of points in one dimension. We develop a dynamic programming algorithm for this case that runs in $O\left(n^{2}\right)$ time.
\end{abstract}

\section{Introduction}

Many interesting data sets can be interpreted as point sets in $\mathbb{R}^{d}$, where the dimension $d$ is the number of features of interest of each data point, and the coordinates are the values of each feature. Given such a data set, graph-based semi-supervised learning is a paradigm for making predictions on the unlabelled data using the proximity among the data points and possibly some labelled data (e.g. [3, 6, 9, 12, 14, 15, 16]). Classification, regression, and clustering are some popular applications. The graph has to be set up first in order to perform the subsequent processing. This requires the determination of the graph edges and the weights to be associated with the edges. For example, let $w_{i j}$ denote the weight determined for the edge that connects two points $p_{i}$ and $p_{j}$, and regression can be performed to predict function values $f_{i}$ 's at the points $p_{i}$ 's by minimizing $\sum_{i, j} w_{i j}\left(f_{i}-f_{j}\right)^{2}$, subject to fixing the subset of known $f_{i}$ 's [3]. To allow efficient data analysis, it is important that the weighted graph is sparse.

The graph connectivity should satisfy the property that similar discrete samples are connected. To this end, different proximity graphs have been suggested for connecting proximal points. The $k N N$-graph connects each point to its $k$ nearest neighbors. The $\varepsilon$-ball graph connects each point to all other points that are within a distance $\varepsilon$. After fixing the graph connectivity, edges to "near" points are given large weights and edges to "far away" points are given small weights. That is, the larger the weight of an edge between points $p$ and $q$, the higher the influence of $q$ on $p$ and vice versa. It is thus inappropriate to use the Euclidean distances among the points as edge weights. Naively setting an edge weight as the reciprocal of the edge length does not work either because the influence of a point is required to fall much more rapidly as that point moves farther away. It has been proposed to associate a weight of $\exp \left(-\ell^{2} / 2 \sigma^{2}\right)$ to an edge of Euclidean length $\ell$ for some a priori determined parameter $\sigma$ (e.g. [12]). A well-tuned

\footnotetext{
${ }^{*}$ SWC is supported by Research Grants Council, Hong Kong, China (project no. 16203718). OC and TL were supported by ICT R\&D program of MSIP/IITP [IITP-2015-0-00199]. A preliminary version appeared in Proceedings of the Canadian Conference on Computational Geometry, 2020 [2].

${ }^{\dagger}$ Department of Computer Science and Engineering, HKUST, Hong Kong. Email: scheng@cse.ust.hk, taegyoung.lee@connect.ust.hk, zrenah@connect.ust.hk.

${ }^{\ddagger}$ Korea Advanced Institute of Science \& Technology (KAIST). Email: otfried@kaist.airpost.net.
} 
$\sigma$ is important. A slight change in $\sigma$ may greatly affect the processing outcomes as observed in some previous work (e.g. [14]). Several studies have found the $k N N$-graph and the $\varepsilon$-ball graphs to be inferior to other proximity graphs [3, 4, 15] for which both the graph connectivity and the edge weights are determined simultaneously by solving an optimization problem.

We consider the graph proposed by Daitch, Kelner, and Spielman [3]. It is provably sparse, and experiments have shown that it offers good performance in classification, clustering and regression. This graph is defined via quadratic optimization as follows: Let $P=\left\{p_{1}, p_{2}, \ldots, p_{n}\right\}$ be a set of $n$ points in $\mathbb{R}^{d}$. We assign weights $w_{i j} \geqslant 0$ to each pair of points $\left(p_{i}, p_{j}\right)$, such that $w_{i j}=w_{j i}$ and $w_{i i}=0$. These weights determine for each point $p_{i}$ a vector $\vec{v}_{i}$, as follows:

$$
\vec{v}_{i}=\sum_{j=1}^{n} w_{i j}\left(p_{j}-p_{i}\right)
$$

Let $v_{i}$ denote $\left\|\vec{v}_{i}\right\|$. The weights are chosen so as to minimize the sum

$$
Q=\sum_{i=1}^{n} v_{i}^{2}
$$

under the constraint that the weights for each point add up to at least one (to prevent the trivial solution of $w_{i j}=0$ for all $i$ and $j$ ):

$$
\sum_{j=1}^{n} w_{i j} \geqslant 1 \quad \text { for } 1 \leqslant i \leqslant n .
$$

The resulting graph contains an edge connecting $p_{i}$ and $p_{j}$ if and only if $w_{i j}>0$.

Daitch et al. [3] showed that there is an optimal solution where at most $(d+1) n$ weights are non-zero. Moreover, in two dimensions, optimal weights can be chosen such that the graph is planar.

The optimal weights can be computed by quadratic programming. A quadratic programming problem with $m$ variables can be solved in $\tilde{O}\left(m^{3}\right)$ time in the worst case [10]. In our case, there are $n(n-1) / 2$ variables, which gives a worst-case running time of $\tilde{O}\left(n^{6}\right)$. Graphs based on optimizing other convex quality measures have also been considered [6, 15]. Our goal is to design an algorithm to compute the optimal weights in Daitch et al.'s formulation that is significantly faster than quadratic programming. Perhaps surprisingly, this problem is challenging even for points in one dimension, that is, when all points lie on a line. In this case, it is not difficult to show (Lemma 2.1) that there is an optimal solution such that $w_{i j}>0$ if and only if $p_{i}$ and $p_{j}$ are consecutive.

Despite its simplicity, the one-dimensional problem can model the task of detecting change points and concept drift in a time series (e.g. [1, 15, 7, 8, 13]); for example, seasonal changes in sales figures and customer behavior. A time series of multi-dimensional data $\left(z_{1}, z_{2}, \cdots\right)$ is given, and the problem is to decide the time steps $t$ at which there is a "significant change" from $z_{t-1}$ to $z_{t}$. Suppose that the "distance" between $z_{t-1}$ and $z_{t}$ can be computed according to some formula appropriate for the application (e.g. [5]). By forming a path graph with vertices corresponding to the data points and edge weights determined as mentioned previously, one can apply clustering algorithms (e.g. [3, 12]) to group "similar" vertices and detect the change points as the boundaries of adjacent clusters. This gives a potential application of the graph fitting problem in one dimension.

In general, although there are only $n-1$ variables in one dimension, the weights in an optimal solution do not seem to follow any simple pattern as we illustrate in the following two examples. 
Some weights in an optimal solution can be arbitrarily high. Consider four points $p_{1}, p_{2}, p_{3}, p_{4}$ in left-to-right order such that $p_{2}-p_{1}=p_{4}-p_{3}=1$ and $p_{3}-p_{2}=\varepsilon$. By symmetry, $w_{12}=w_{34}$, and so $v_{1}=v_{4}=w_{12}$. Since $w_{12}+w_{23} \geqslant 1$ and $w_{23}+w_{34} \geqslant 1$ are trivially satisfied by the requirement that $w_{12}=w_{34} \geqslant 1$, we can make $v_{2}$ zero by setting $w_{23}=w_{12} / \varepsilon$. In the optimal solution, $w_{12}=w_{34}=1$ and $w_{23}=1 / \varepsilon$. So $w_{23}$ can be arbitrarily large.

Given points $p_{1}, \cdots, p_{n}$ in left-to-right order, it seems ideal to make $v_{i}$ a zero vector. One can do this for $i \in[2, n-1]$ by setting $w_{i-1, i} / w_{i, i+1}=\left(p_{i+1}-p_{i}\right) /\left(p_{i}-p_{i-1}\right)$, however, some of the constraints $w_{i}+w_{i+1} \geqslant 1$ may be violated. Even if we are lucky that for $i \in[2, n-1]$, we can set $w_{i-1, i} / w_{i, i+1}=\left(p_{i+1}-p_{i}\right) /\left(p_{i}-p_{i-1}\right)$ without violating $w_{i}+w_{i+1} \geqslant 1$, the solution may not be optimal as we show below. Requiring $v_{i}=0$ for $i \in[2, n-1]$ gives $v_{1}=v_{n}=w_{12}\left(p_{2}-p_{1}\right)$. In general, we have $p_{2}-p_{1} \neq p_{n}-p_{n-1}$, so we can assume that $p_{2}-p_{1}>p_{n}-p_{n-1}$. Then, $w_{n-1, n}=w_{12}\left(p_{2}-p_{1}\right) /\left(p_{n}-p_{n-1}\right)>1$ as $w_{12} \geqslant 1$. Since $w_{n-1, n}>1$, one can decrease $w_{n-1, n}$ by a small quantity $\delta$ while keeping its value greater than 1 . Both constraints $w_{n-1, n} \geqslant 1$ and $w_{n-2, n-1}+w_{n-1, n} \geqslant 1$ are still satisfied. Observe that $v_{n}$ drops to $w_{12}\left(p_{2}-p_{1}\right)-\delta\left(p_{n}-p_{n-1}\right)$ and $v_{n-1}$ increases to $\delta\left(p_{n}-p_{n-1}\right)$. Hence, $v_{n-1}^{2}+v_{n}^{2}$ decreases by $2 \delta w_{12}\left(p_{2}-p_{1}\right)\left(p_{n}-p_{n-1}\right)-$ $2 \delta^{2}\left(p_{n}-p_{n-1}\right)^{2}$, and so does $Q$. The original setting of the weights is thus not optimal. If $w_{n-3, n-2}+w_{n-2, n-1}>1$, it will bring further benefit to decrease $w_{n-2, n-1}$ slightly so that $v_{n-1}$ decreases slightly from $\delta\left(p_{n}-p_{n-1}\right)$ and $v_{n-2}$ increases slightly from zero. Intuitively, instead of concentrating $w_{12}\left(p_{2}-p_{1}\right)$ at $v_{n}$, it is better to distribute it over multiple points in order to decrease the sum of squares. But it does not seem easy to determine the best weights.

Although there are only $n-1$ variables in one dimension, quadratic programming still yields a running time of $\tilde{O}\left(n^{3}\right)$. We present a dynamic programming algorithm that computes the optimal weights in $O\left(n^{2}\right)$ time in the one-dimensional case. The intermediate solutions computed by the algorithm have an interesting structure where the derivative of the quality measure depends on the derivative of a subproblem's quality measure as well as the inverse of this derivative function. We have experimental evidence that explicitly representing the intermediate solutions may require exponential time and space, and so we develop an implicit representation that facilitates the dynamic programming algorithm.

\section{A single-parameter quality measure function}

We will assume that the points are given in sorted order, so that $p_{1}<p_{2}<p_{3}<\cdots<p_{n}$. We first argue that the only weights that need to be non-zero are the weights between consecutive points, that is, weights of the form $w_{i, i+1}$.

Lemma 2.1. For $d=1$, there is an optimal solution where only weights between consecutive points are non-zero.

Proof. For a solution $S$ and $t \in\{1,2, \ldots, n-1\}$, let $z_{t}(S)$ be the number of non-zero weights at distance $t$ (that is, $z_{t}(S)$ is the cardinality of the set $\left\{i \mid w_{i, i+t}>0\right.$ in $\left.S\right\}$ ), and let $\mathbf{z}(S)$ be the vector

$$
\mathbf{z}(S)=\left(z_{n-1}(S), z_{n-2}(S), \ldots, z_{3}(S), z_{2}(S)\right) .
$$

Among all optimal solutions, that is, solutions minimizing the quality $Q$, let $O$ be a solution that lexicographically minimizes $\mathbf{z}(O)$. We will show that $\mathbf{z}(O)=(0, \ldots, 0)$.

Assume to the contrary that there is a weight $w_{i, k}>0$ for some $i<k-1$ in $O$. Let $j$ be an arbitrary index strictly between $i$ and $k$. We construct a new optimal solution as follows: Let $a=p_{j}-p_{i}, b=p_{k}-p_{j}$, and $w=w_{i, k}$. In the new solution, we set $w_{i, k}=0$, increase $w_{i, j}$ by $\frac{a+b}{a} w$, and increase $w_{j, k}$ by $\frac{a+b}{b} w$. Note that since $a+b>a$ and $a+b>b$, the sum of weights at each vertex increases, and so the weight vector remains feasible. The value $v_{j}$ changes by 
$-a \times \frac{a+b}{a} w+b \times \frac{a+b}{b} w=0$; the value $v_{i}$ changes by $-(a+b) \times w+a \times \frac{a+b}{a} w=0$; the value $v_{k}$ changes by $+(a+b) \times w-b \times \frac{a+b}{b} w=0$. It follows that the new solution $O^{\prime}$ has the same quality as $O$, and is therefore also optimal. Since $\mathbf{z}\left(O^{\prime}\right)$ is lexicographically smaller than $\mathbf{z}(O)$, this is a contradiction to the choice of $O$.

To simplify the notation, we set $d_{i}=p_{i+1}-p_{i}$, for $1 \leqslant i<n$; rename the weights as $w_{i}:=w_{i, i+1}$, again for $1 \leqslant i<n$; observe that

$$
\begin{aligned}
v_{1} & =w_{1} d_{1}, \\
v_{i} & =\left|w_{i} d_{i}-w_{i-1} d_{i-1}\right| \quad \text { for } 2 \leqslant i \leqslant n-1, \\
v_{n} & =w_{n-1} d_{n-1} .
\end{aligned}
$$

For $i \in[2, n-1]$, we introduce the quantity

$$
\begin{aligned}
Q_{i} & =d_{i}^{2} w_{i}^{2}+\sum_{j=1}^{i} v_{j}^{2} \\
& =d_{i}^{2} w_{i}^{2}+d_{1}^{2} w_{1}^{2}+\sum_{j=2}^{i}\left(d_{j} w_{j}-d_{j-1} w_{j-1}\right)^{2}
\end{aligned}
$$

and note that $Q_{n-1}=\sum_{i=1}^{n} v_{i}^{2}=Q$. Thus, our goal is to choose the $n-1$ non-negative weights $w_{1}, \ldots, w_{n-1}$ such that $Q_{n-1}$ is minimized, under the constraints

$$
\begin{aligned}
w_{1} & \geqslant 1, \\
w_{j}+w_{j+1} & \geqslant 1 \\
w_{n-1} & \geqslant 1 .
\end{aligned} \quad \text { for } 2 \leqslant j \leqslant n-2,
$$

The quantity $Q_{i}$ depends on $w_{1}, w_{2}, \ldots, w_{i}$. We concentrate on $w_{i}$ and consider the function

$$
w_{i} \mapsto Q_{i}\left(w_{i}\right)=\min _{w_{1}, \ldots, w_{i-1}} Q_{i}\left(w_{1}, w_{2}, \ldots, w_{i-1}, w_{i}\right),
$$

where the minimum is taken over all choices of $w_{1}, \ldots, w_{i-1}$ that respect the constraints $w_{1} \geqslant 1$ and $w_{j}+w_{j+1} \geqslant 1$ for $2 \leqslant j \leqslant i-1$. The function $Q_{i}\left(w_{i}\right)$ is defined on $[0, \infty)$.

We denote the derivative of the function $w_{i} \mapsto Q_{i}\left(w_{i}\right)$ by $R_{i}$. We will see shortly that $R_{i}$ is a continuous, piecewise linear function. Since $R_{i}$ is not differentiable everywhere, we define $S_{i}(x)$ to be the right derivative of $R_{i}$, that is

$$
S_{i}(x)=\lim _{y \rightarrow x^{+}} R_{i}^{\prime}(y) .
$$

The following result discusses $R_{i}$ and $S_{i}$. The shorthand

$$
\xi_{i}:=2 d_{i} d_{i+1}, \quad \text { for } 1 \leqslant i<n-1,
$$

will be convenient in its proof and the rest of the paper.

Theorem 2.1. The function $R_{i}$ is strictly increasing, continuous, and piecewise linear on the range $[0, \infty)$. We have $R_{i}(0)<0, S_{i}(x) \geqslant(2+2 / i) d_{i}^{2}$ for all $x \geqslant 0$, and $R_{i}(x)=(2+2 / i) d_{i}^{2} x$ for sufficiently large $x>0$. 
Proof. We prove all claims by induction over $i$. The base case is $i=2$. Observe that

$$
Q_{2}=v_{1}^{2}+v_{2}^{2}+d_{2}^{2} w_{2}^{2}=2 d_{1}^{2} w_{1}^{2}-2 d_{1} d_{2} w_{1} w_{2}+2 d_{2}^{2} w_{2}^{2} .
$$

The derivative with respect to $w_{1}$ is

$$
\frac{\partial}{\partial w_{1}} Q_{2}=4 d_{1}^{2} w_{1}-2 d_{1} d_{2} w_{2}
$$

which implies that $Q_{2}$ is minimized for $w_{1}=\frac{d_{2}}{2 d_{1}} w_{2}$. This choice is feasible (with respect to the constraint $w_{1} \geqslant 1$ ) when $w_{2} \geqslant \frac{2 d_{1}}{d_{2}}$. If $w_{2}<\frac{2 d_{1}}{d_{2}}$, then $\frac{\partial}{\partial w_{1}} Q_{2}$ is positive for all values of $w_{1} \geqslant 1$, so the minimum occurs at $w_{1}=1$. It follows that

$$
Q_{2}\left(w_{2}\right)= \begin{cases}\frac{3}{2} d_{2}^{2} w_{2}^{2} & \text { for } w_{2} \geqslant \frac{2 d_{1}}{d_{2}} \\ 2 d_{2}^{2} w_{2}^{2}-\xi_{1} w_{2}+2 d_{1}^{2} & \text { otherwise }\end{cases}
$$

and so we have

$$
R_{2}\left(w_{2}\right)= \begin{cases}3 d_{2}^{2} w_{2} & \text { for } w_{2} \geqslant \frac{2 d_{1}}{d_{2}} \\ 4 d_{2}^{2} w_{2}-\xi_{1} & \text { otherwise. }\end{cases}
$$

In other words, $R_{2}$ is piecewise linear and has a single breakpoint at $\frac{2 d_{1}}{d_{2}}$. The function $R_{2}$ is continuous because $3 d_{2}^{2} w_{2}=4 d_{2}^{2} w_{2}-\xi_{1}$ when $w_{2}=\frac{2 d_{1}}{d_{2}}$. We have $R_{2}(0)=-\xi_{1}<0, S_{2}(x) \geqslant 3 d_{2}^{2}$ for all $x \geqslant 0$, and $R_{2}(x)=3 d_{2}^{2} x$ for $x \geqslant \frac{2 d_{1}}{d_{2}}$. The fact that $S_{2}(x) \geqslant 3 d_{2}^{2}>0$ makes $R_{2}$ strictly increasing.

Consider now $i \geqslant 2$, assume that $R_{i}$ and $S_{i}$ satisfy the induction hypothesis, and consider $Q_{i+1}$. By definition, we have

$$
Q_{i+1}=Q_{i}-\xi_{i} w_{i} w_{i+1}+2 d_{i+1}^{2} w_{i+1}^{2} .
$$

For a given value of $w_{i+1} \geqslant 0$, we need to find the value of $w_{i}$ that will minimize $Q_{i+1}$. The derivative is

$$
\frac{\partial}{\partial w_{i}} Q_{i+1}=R_{i}\left(w_{i}\right)-\xi_{i} w_{i+1} .
$$

The minimum thus occurs when $R_{i}\left(w_{i}\right)=\xi_{i} w_{i+1}$.

Since $R_{i}$ is a strictly increasing continuous function with $R_{i}(0)<0$ and $\lim _{x \rightarrow \infty} R_{i}(x)=\infty$, for any given $w_{i+1} \geqslant 0$, there exists a unique value $w_{i}=R_{i}^{-1}\left(\xi_{i} w_{i+1}\right)$. However, we also need to satisfy the constraint $w_{i}+w_{i+1} \geqslant 1$.

We first show that $R_{i+1}$ is continuous and piecewise linear, and that $R_{i+1}(0)<0$. We will distinguish two cases, based on the value of $w_{i}^{\circ}:=R_{i}^{-1}(0)$.

Case 1: $w_{i}^{\circ} \geqslant 1$. This means that $R_{i}^{-1}\left(\xi_{i} w_{i+1}\right) \geqslant 1$ for any $w_{i+1} \geqslant 0$, and so the constraint of $w_{i}+w_{i+1} \geqslant 1$ is satisfied for the optimal choice of $w_{i}=R_{i}^{-1}\left(\xi_{i} w_{i+1}\right)$. It follows that

$$
\begin{aligned}
Q_{i+1}\left(w_{i+1}\right)= & Q_{i}\left(R_{i}^{-1}\left(\xi_{i} w_{i+1}\right)\right)-\xi_{i} w_{i+1} R_{i}^{-1}\left(\xi_{i} w_{i+1}\right) \\
& +2 d_{i+1}^{2} w_{i+1}^{2} .
\end{aligned}
$$


The derivative $R_{i+1}$ is therefore

$$
\begin{aligned}
R_{i+1}\left(w_{i+1}\right)= & R_{i}\left(R_{i}^{-1}\left(\xi_{i} w_{i+1}\right)\right) \frac{\xi_{i}}{R_{i}^{\prime}\left(R_{i}^{-1}\left(\xi_{i} w_{i+1}\right)\right)} \\
& -\xi_{i} R_{i}^{-1}\left(\xi_{i} w_{i+1}\right) \\
& -\xi_{i} w_{i+1} \frac{\xi_{i}}{R_{i}^{\prime}\left(R_{i}^{-1}\left(\xi_{i} w_{i+1}\right)\right)} \\
& +4 d_{i+1}^{2} w_{i+1} \\
= & 4 d_{i+1}^{2} w_{i+1}-\xi_{i} R_{i}^{-1}\left(\xi_{i} w_{i+1}\right) .
\end{aligned}
$$

Since $R_{i}$ is continuous and piecewise linear, so is $R_{i}^{-1}$, and therefore $R_{i+1}$ is continuous and piecewise linear. We have $R_{i+1}(0)=-\xi_{i} w_{i}^{\circ}<0$.

Case 2: $w_{i}^{\circ}<1$. Consider the function $x \mapsto f(x)=x+R_{i}(x) / \xi_{i}$. Since $R_{i}$ is continuous and strictly increasing by the inductive assumption, so is the function $f$. Observe that $f\left(w_{i}^{\circ}\right)=$ $w_{i}^{\circ}<1$. As $w_{i}^{\circ}<1$, we have $R_{i}(1)>R_{i}\left(w_{i}^{\circ}\right)=0$, which implies that $f(1)>1$. Thus, there exists a unique value $w_{i}^{\bowtie} \in\left(w_{i}^{\circ}, 1\right)$ such that $f\left(w_{i}^{\bowtie}\right)=w_{i}^{\bowtie}+R_{i}\left(w_{i}^{\bowtie}\right) / \xi_{i}=1$.

For $w_{i+1} \geqslant 1-w_{i}^{\bowtie}=R_{i}\left(w_{i}^{\bowtie}\right) / \xi_{i}$, we have $R_{i}^{-1}\left(\xi_{i} w_{i+1}\right) \geqslant w_{i}^{\bowtie}$, and so $R_{i}^{-1}\left(\xi_{i} w_{i+1}\right)+w_{i+1} \geqslant 1$. This implies that the constraint $w_{i}+w_{i+1} \geqslant 1$ is satisfied when $Q_{i+1}\left(w_{i+1}\right)$ is minimized for the optimal choice of $w_{i}=R_{i}^{-1}\left(\xi_{i} w_{i+1}\right)$. So $R_{i+1}$ is as in (4) in Case 1.

When $w_{i+1}<1-w_{i}^{\bowtie}$, the constraint $w_{i}+w_{i+1} \geqslant 1$ implies that $w_{i} \geqslant 1-w_{i+1}>w_{i}^{\bowtie}$. For any $w_{i}>w_{i}^{\bowtie}$ we have $\frac{\partial}{\partial w_{i}} Q_{i+1}=R_{i}\left(w_{i}\right)-\xi_{i} w_{i+1}>R_{i}\left(w_{i}^{\bowtie}\right)-\xi_{i}\left(1-w_{i}^{\bowtie}\right)=0$. So $Q_{i+1}$ is increasing, and the minimal value is obtained for the smallest feasible choice of $w_{i}$, that is, for $w_{i}=1-w_{i+1}$. It follows that

$$
\begin{aligned}
Q_{i+1}\left(w_{i+1}\right)= & Q_{i}\left(1-w_{i+1}\right)-\xi_{i} w_{i+1}\left(1-w_{i+1}\right) \\
& +2 d_{i+1}^{2} w_{i+1}^{2} \\
= & Q_{i}\left(1-w_{i+1}\right)-\xi_{i} w_{i+1} \\
& +\left(\xi_{i}+2 d_{i+1}^{2}\right) w_{i+1}^{2}
\end{aligned}
$$

and so the derivative $R_{i+1}$ is

$$
\begin{aligned}
R_{i+1}\left(w_{i+1}\right)= & -R_{i}\left(1-w_{i+1}\right) \\
& +\left(2 \xi_{i}+4 d_{i+1}^{2}\right) w_{i+1}-\xi_{i} .
\end{aligned}
$$

Combining (4) and (5), we have

- If $w_{i+1}<1-w_{i}^{\bowtie}$, then

$$
\begin{aligned}
R_{i+1}\left(w_{i+1}\right)= & -R_{i}\left(1-w_{i+1}\right) \\
& +\left(2 \xi_{i}+4 d_{i+1}^{2}\right) w_{i+1}-\xi_{i} .
\end{aligned}
$$

- If $w_{i+1} \geqslant 1-w_{i}^{\bowtie}$, then

$$
R_{i+1}\left(w_{i+1}\right)=4 d_{i+1}^{2} w_{i+1}-\xi_{i} R_{i}^{-1}\left(\xi_{i} w_{i+1}\right) .
$$


For $w_{i+1}=1-w_{i}^{\bowtie}$, we have $R_{i}\left(1-w_{i+1}\right)=R_{i}\left(w_{i}^{\bowtie}\right)=\xi_{i}\left(1-w_{i}^{\bowtie}\right)$ and $R_{i}^{-1}\left(\xi_{i} w_{i+1}\right)=$ $R_{i}^{-1}\left(\xi_{i}\left(1-w_{i}^{\bowtie}\right)\right)=w_{i}^{\bowtie}$, and so both expressions have the same value:

$$
\begin{aligned}
& -R_{i}\left(1-w_{i+1}\right)+\left(2 \xi_{i}+4 d_{i+1}^{2}\right) w_{i+1}-\xi_{i} \\
= & \xi_{i} w_{i}^{\bowtie}-\xi_{i}+2 \xi_{i}-2 \xi_{i} w_{i}^{\bowtie}+4 d_{i+1}^{2}\left(1-w_{i}^{\bowtie}\right)-\xi_{i} \\
= & 4 d_{i+1}^{2}\left(1-w_{i}^{\bowtie}\right)-\xi_{i} w_{i}^{\bowtie} \\
= & 4 d_{i+1}^{2}\left(1-w_{i}^{\bowtie}\right)-\xi_{i} R_{i}^{-1}\left(\xi_{i} w_{i+1}\right) .
\end{aligned}
$$

Since $R_{i}$ is continuous and piecewise linear, this implies that $R_{i+1}$ is continuous and piecewise linear. We have $R_{i+1}(0)=-R_{i}(1)-\xi_{i}$. Since $w_{i}^{\circ}<1$, we have $R_{i}(1)>R_{i}\left(w_{i}^{\circ}\right)=0$, and so $R_{i+1}(0)<0$.

Next, we show that $S_{i+1}(x) \geqslant(2+2 / i+1) d_{i+1}^{2}$ for all $x \geqslant 0$, which implies that $R_{i+1}$ is strictly increasing. If $w_{i}^{\circ}<1$ and $x<1-w_{i}^{\bowtie}$, then by (6) ,

$$
\begin{aligned}
S_{i+1}(x)= & S_{i}(1-x)+2 \xi_{i}+4 d_{i+1}^{2} \\
& >4 d_{i+1}^{2} \\
& >(2+2 / i+1) d_{i+1}^{2} .
\end{aligned}
$$

If $w_{i}^{\circ} \geqslant 1$ or $x>1-w_{i}^{\bowtie}$, we have by (4) and (7) that $R_{i+1}(x)=4 d_{i+1}^{2} x-\xi_{i} R_{i}^{-1}\left(\xi_{i} x\right)$. By the inductive assumption that $S_{i}(x) \geqslant(2+2 / i) d_{i}^{2}$ for all $x \geqslant 0$, we get $\frac{\partial}{\partial x} R_{i}^{-1}(x) \leqslant 1 /\left((2+2 / i) d_{i}^{2}\right)$. It follows that

$$
\begin{aligned}
S_{i+1}(x) & \geqslant 4 d_{i+1}^{2}-\frac{\left(2 d_{i} d_{i+1}\right)^{2}}{(2+2 / i) d_{i}^{2}}=\left(4-\frac{4}{2+2 / i}\right) d_{i+1}^{2} \\
& =\left(4-\frac{2 i}{i+1}\right) d_{i+1}^{2} \\
& =\left(2+\frac{2}{i+1}\right) d_{i+1}^{2} .
\end{aligned}
$$

This establishes the lower bound on $S_{i+1}(x)$.

Finally, by the inductive assumption, when $x$ is large enough, we have $R_{i}^{-1}(x)=x /((2+$ $\left.2 / i) d_{i}^{2}\right)$, and so

$$
\begin{aligned}
R_{i+1}(x) & =4 d_{i+1}^{2} x-\frac{\left(2 d_{i} d_{i+1}\right)^{2}}{(2+2 / i) d_{i}^{2}} x \\
& =\left(2+\frac{2}{i+1}\right) d_{i+1}^{2} x,
\end{aligned}
$$

completing the inductive step and therefore the proof.

\section{The algorithm}

Our algorithm progressively constructs a representation of the functions $R_{2}, R_{3}, \ldots, R_{n-1}$. The function representation supports the following three operations:

- Op 1: given $x$, return $R_{i}(x)$;

- Op 2: given $y$, return $R_{i}^{-1}(y)$; 
- Op 3: given $\xi$, return $x^{\bowtie}$ such that $x^{\bowtie}+\frac{R_{i}\left(x^{\infty}\right)}{\xi}=1$.

The proof of Theorem 2.1 gives the relation between $R_{i+1}$ and $R_{i}$. This will allow us to construct the functions one by one - we discuss the detailed implementation in Sections 3.1 and 3.2 below.

Once all functions $R_{2}, \ldots, R_{n-1}$ are constructed, the optimal weights $w_{1}, w_{2}, \ldots, w_{n-1}$ are computed from the $R_{i}$ 's as follows. Recall that $Q=Q_{n-1}$, so $w_{n-1}$ is the value minimizing $Q_{n-1}\left(w_{n-1}\right)$ under the constraint $w_{n-1} \geqslant 1$. If $R_{n-1}^{-1}(0) \geqslant 1$, then $R_{n-1}^{-1}(0)$ is the optimal value for $w_{n-1}$; otherwise, we set $w_{n-1}$ to 1 .

To obtain $w_{n-2}$, recall from (3) that $Q=Q_{n-1}=Q_{n-2}\left(w_{n-2}\right)-\xi_{n-2} w_{n-2} w_{n-1}+2 d_{n-1}^{2} w_{n-1}^{2}$. Since we have already determined the correct value of $w_{n-1}$, it remains to choose $w_{n-2}$ so that $Q_{n-1}$ is minimized. Since

$$
\frac{\partial}{\partial w_{n-2}} Q_{n-1}=R_{n-2}\left(w_{n-2}\right)-\xi_{n-2} w_{n-1},
$$

$Q_{n-1}$ is minimized when $R_{n-2}\left(w_{n-2}\right)=\xi_{n-2} w_{n-1}$, and so $w_{n-2}=R_{n-2}^{-1}\left(\xi_{n-2} w_{n-1}\right)$.

In general, for $i \in[2, n-2]$, we can obtain $w_{i}$ from $w_{i+1}$ by observing that

$$
Q_{n-1}=Q_{i}\left(w_{i}\right)-\xi_{i} w_{i} w_{i+1}+g\left(w_{i+1}, \ldots, w_{n-1}\right)
$$

where $g$ is function that only depends on $w_{i+1}, \ldots, w_{n-1}$. Taking the derivative again, we have

$$
\frac{\partial}{\partial w_{i}} Q_{n-1}=R_{i}\left(w_{i}\right)-\xi_{i} w_{i+1}
$$

so choosing $w_{i}=R_{i}^{-1}\left(\xi_{i} w_{i+1}\right)$ minimizes $Q_{n-1}$. To also satisfy the constraint $w_{i}+w_{i+1} \geqslant 1$, we need to choose $w_{i}=\max \left\{R_{i}^{-1}\left(\xi_{i} w_{i+1}\right), 1-w_{i+1}\right\}$ for $i \in[2, n-2]$. Finally, from the discussion that immediately follows (1), we set $w_{1}=\max \left\{\frac{d_{2}}{2 d_{1}} w_{2}, 1\right\}$. To summarize, we have

$$
\begin{aligned}
w_{n-1} & =\max \left\{R_{n-1}^{-1}(0), 1\right\}, \\
w_{i} & =\max \left\{R_{i}^{-1}\left(\xi_{i} w_{i+1}\right), 1-w_{i+1}\right\}, \text { for } i \in[2, n-2], \\
w_{1} & =\max \left\{\frac{d_{2}}{2 d_{1}} w_{2}, 1\right\} .
\end{aligned}
$$

It follows that we can obtain the optimal weights using a single Op 2 on each $R_{i}$.

\subsection{Explicit representation of piecewise linear functions}

Since $R_{i}$ is a piecewise linear function, a natural representation is a sequence of linear functions, together with the sequence of breakpoints. Since $R_{i}$ is strictly increasing, all three operations can then be implemented to run in time $O(\log k)$ using binary search, where $k$ is the number of function pieces.

We construct the functions $R_{i}$, for $i=2, \ldots, n-1$, one by one.

The function $R_{2}$ consists of exactly two pieces. We construct it directly from $d_{1}, d_{2}$, and $\xi_{1}$ using (2).

To construct $R_{i+1}$, we make use of the explicit representation of $R_{i}$ that we have already computed. We first compute $w_{i}^{\circ}=R_{i}^{-1}(0)$ using Op 2 on $R_{i}$. If $w_{i}^{\circ} \geqslant 1$, then by (4) each piece of $R_{i}$, starting at the $x$-coordinate $w_{i}^{\circ}$, gives rise to a linear piece of $R_{i+1}$, so the number of pieces of $R_{i+1}$ is at most that of $R_{i}$.

If $w_{i}^{\circ}<1$, then we compute $w_{i}^{\bowtie}$ using Op 3 on $R_{i}$. The new function $R_{i+1}$ has a breakpoint at $1-w_{i}^{\bowtie}$ by (6) and (7). Its pieces for $x \geqslant 1-w_{i}^{\bowtie}$ are computed from the pieces of $R_{i}$ starting 
at the $x$-coordinate $w_{i}^{\bowtie}$. Its pieces for $0 \leqslant x<1-w_{i}^{\bowtie}$ are computed from the pieces of $R_{i}$ between the $x$-coordinates 1 and $w_{i}^{\bowtie}$. (Increasing $w_{i+1}$ now corresponds to a decreasing $w_{i}$.)

Since a piece of $R_{i}$ that covers $x$-coordinates in the range $\left[w_{i}^{\bowtie}, 1\right]$ gives rise to two pieces of $R_{i+1}$, the number of pieces of $R_{i+1}$ can be twice the number of pieces of $R_{i}$. If this case occurs in every step of the construction, then function $R_{i}$ will have $2^{i-1}$ pieces, leading to exponential time and space complexity.

It is hard to manually construct inputs that exhibit exponential growth in the number of pieces of $R_{i}$. We have therefore used the Z3 theorem prover [11] to try and satisfy the constraints under which $R_{n-1}$ has $2^{n-2}$ pieces. For $n=10$, which is the largest instance we have been able to solve using Z3, we obtain the following nine distances:

$\begin{array}{ccccccccc}d_{1} & d_{2} & d_{3} & d_{4} & d_{5} & d_{6} & d_{7} & d_{8} & d_{9} \\ 11569 & 49184 & 65536 & 98304 & 109056 & 145408 & 146432 & 147456 & 32768\end{array}$

We have implemented the algorithm and verified using exact arithmetic that for these distances, the function $R_{i}$ indeed has $2^{i-1}$ pieces, for $2 \leqslant i \leqslant 9$.

Based on this experiment, we find it unlikely that the explicit representation of the functions $R_{i}$ will lead to a polynomial-time algorithm.

\subsection{A quadratic time implementation}

To guarantee a polynomial running time, we turn to an implicit representation of $R_{i}$. This representation is based on the fact that there is a linear relationship between points on the graphs of the functions $R_{i}$ and $R_{i+1}$. Concretely, let $y_{i}=R_{i}\left(x_{i}\right)$, and $y_{i+1}=R_{i+1}\left(x_{i+1}\right)$. Recall the following relation from (4) for the case of $w_{i}^{\circ} \geqslant 1$ :

$$
R_{i+1}\left(w_{i+1}\right)=4 d_{i+1}^{2} w_{i+1}-\xi_{i} R_{i}^{-1}\left(\xi_{i} w_{i+1}\right) .
$$

We can express this relation as a system of two equations:

$$
\begin{aligned}
y_{i+1} & =4 d_{i+1}^{2} x_{i+1}-\xi_{i} x_{i}, \\
y_{i} & =\xi_{i} x_{i+1} .
\end{aligned}
$$

This can be rewritten as

$$
\begin{aligned}
& y_{i+1}=4 d_{i+1}^{2} y_{i} / \xi_{i}-\xi_{i} x_{i}, \\
& x_{i+1}=y_{i} / \xi_{i},
\end{aligned}
$$

or in matrix notation

$$
\left(\begin{array}{c}
x_{i+1} \\
y_{i+1} \\
1
\end{array}\right)=M_{i+1} \cdot\left(\begin{array}{c}
x_{i} \\
y_{i} \\
1
\end{array}\right)
$$

where

$$
M_{i+1}=\left(\begin{array}{ccc}
0 & 1 / \xi_{i} & 0 \\
-\xi_{i} & 4 d_{i+1}^{2} / \xi_{i} & 0 \\
0 & 0 & 1
\end{array}\right)
$$

On the other hand, if $w_{i}^{\circ}<1$, then $R_{i+1}$ has a breakpoint at $1-w_{i}^{\bowtie}$. The value $w_{i}^{\bowtie}$ can be obtained by appying Op 3 to $R_{i}$. We compute the coordinates of this breakpoint: $\left(1-w_{i}^{\bowtie}, R_{i+1}\left(1-w_{i}^{\bowtie}\right)\right)$. Note that $R_{i+1}\left(1-w_{i}^{\bowtie}\right)=4 d_{i+1}^{2}\left(1-w_{i}^{\bowtie}\right)-\xi_{i} R_{i}^{-1}\left(\xi_{i}\left(1-w_{i}^{\bowtie}\right)\right)$ which 
can be computed by applying Op 2 to $R_{i}$. For $x_{i+1}>1-w_{i}^{\bowtie}$, the relationship between $\left(x_{i}, y_{i}\right)$ and $\left(x_{i+1}, y_{i+1}\right)$ is given by (8). For $0 \leqslant x_{i+1}<1-w_{i}^{\bowtie}$, recall from (5) that

$$
\begin{aligned}
R_{i+1}\left(w_{i+1}\right)= & -R_{i}\left(1-w_{i+1}\right) \\
& +\left(2 \xi_{i}+4 d_{i+1}^{2}\right) w_{i+1}-\xi_{i} .
\end{aligned}
$$

We again rewrite this as

$$
\begin{aligned}
y_{i+1} & =-y_{i}+\left(2 \xi_{i}+4 d_{i+1}^{2}\right) x_{i+1}-\xi_{i}, \\
x_{i} & =1-x_{i+1}
\end{aligned}
$$

which gives

$$
\begin{aligned}
& y_{i+1}=-y_{i}+\left(2 \xi_{i}+4 d_{i+1}^{2}\right)\left(1-x_{i}\right)-\xi_{i}, \\
& x_{i+1}=1-x_{i},
\end{aligned}
$$

or in matrix notation:

$$
\left(\begin{array}{c}
x_{i+1} \\
y_{i+1} \\
1
\end{array}\right)=L_{i+1} \cdot\left(\begin{array}{c}
x_{i} \\
y_{i} \\
1
\end{array}\right)
$$

where

$$
L_{i+1}=\left(\begin{array}{ccc}
-1 & 0 & 1 \\
-2 \xi_{i}-4 d_{i+1}^{2} & -1 & \xi_{i}+4 d_{i+1}^{2} \\
0 & 0 & 1
\end{array}\right) .
$$

We will make use of this relationship to store the function $R_{i+1}$, for $i \geqslant 2$, by storing the breakpoint $\left(x_{i+1}^{*}, y_{i+1}^{*}\right)=\left(1-w_{i}^{\bowtie}, R_{i+1}\left(1-w_{i}^{\bowtie}\right)\right)$ as well as the two matrices $L_{i+1}$ and $M_{i+1}$. The function $R_{2}$ is simply stored explicitly.

We now discuss how the three operations Op 1, Op 2, and Op 3 are implemented on this representation of a function $R_{i}$. For an operation on $R_{i}$, we progressively build transformation matrices $T_{i}^{i}, T_{i-1}^{i}, T_{i-2}^{i}, \ldots, T_{3}^{i}, T_{2}^{i}$ such that $\left(x_{i}, y_{i}, 1\right)=T_{j}^{i} \times\left(x_{j}, y_{j}, 1\right)$ for every $2 \leqslant j \leqslant i$ in a neighborhood of the query. Once we obtain $T_{2}^{i}$, we use our explicit representation of $R_{2}$ to express $y_{i}$ as a linear function of $x_{i}$ in a neighborhood of the query, which then allows us to answer the query.

The first matrix $T_{i}^{i}$ is the identity matrix. We obtain $T_{j}^{i}$ from $T_{j+1}^{i}$, for $j \in[2, i-1]$, as follows: If $R_{j+1}$ has no breakpoint, then $T_{j}^{i}=T_{j+1}^{i} \cdot M_{j+1}$. If $R_{j+1}$ has a breakpoint $\left(x_{j+1}^{*}, y_{j+1}^{*}\right)$, then either $T_{j}^{i}=T_{j+1}^{i} \cdot M_{j+1}$ or $T_{j}^{i}=T_{j+1}^{i} \cdot L_{j+1}$, depending on which side of the breakpoint applies to the answer of the query. We can decide this by comparing $\left(x^{\prime}, y^{\prime}, 1\right)^{t}=T_{j+1}^{i} \cdot\left(x_{j+1}^{*}, y_{j+1}^{*}, 1\right)^{t}$ with the query. More precisely, for Op 1 we compare the input $x$ with $x^{\prime}$, for Op 2 we compare the input $y$ with $y^{\prime}$, and for Op 3 we compute $x^{\prime}+y^{\prime} / \xi$ and compare with 1 .

Assuming the Real-RAM model common in computational geometry, where arithmetic on real numbers takes constant time, it follows that the implicit representation of $R_{i}$ supports all three operations on $R_{i}$ in time $O(i)$.

Finally, we discuss how the representation of all functions $R_{i}$ is obtained. We again build it iteratively, constructing $R_{2}, R_{3}, R_{4}, \ldots, R_{n-1}$, one-by-one in this order. The first function $R_{2}$ is stored explicitly. To construct the implicit representation of $R_{i+1}$, we only need to perform on our representation of $R_{i}$ (that we already computed) one Op 2 to get $w_{i}^{\circ}=R_{i}^{-1}(0)$, one Op 3 to get $w_{i}^{\bowtie}$, and one Op 2 to get $R_{i}^{-1}\left(\xi_{i}\left(1-w_{i}^{\bowtie}\right)\right)$, which allows us to determine the breakpoint $\left(1-w_{i}^{\bowtie}, R_{i+1}\left(1-w_{i}^{\bowtie}\right)\right.$ ), if there is one (when $w_{i}^{\circ}<1$ ). The two matrices $L_{i+1}$ and $R_{i+1}$ can be computed in $O(1)$ time.

Since operations on $R_{i}$ take time $O(i)$, the total time to construct $R_{n-1}$ is $O\left(n^{2}\right)$. 
Theorem 3.1. Given $n$ points on a line, we can compute an optimal set of weights for minimizing the quality measure $Q$ in $O\left(n^{2}\right)$ time under the Real-RAM model.

\section{Conclusion}

We do not have a polynomial time bound on the running time using the explicit representation of the functions $R_{i}$, and our experiments with the Z3 solver suggest that the complexity of $R_{i}$ may indeed increase exponentially with $i$. It would be interesting to determine whether one can construct such a worst-case example for any $n$.

It would also be nice to obtain an algorithm for higher dimensions that is not based on a quadratic programming solver.

In two dimensions, we have conducted some experiments that indicate that the Delaunay triangulation of the point set contains a well-fitting graph. If we choose the graph edges only from the Delaunay edges and compute the optimal edge weights, the resulting quality measure is very close to the best quality measure in the unrestricted case. It is conceivable that one can obtain a provably good approximation from the Delaunay triangulation.

\section{References}

[1] S. Aminikhanghahi and D.J. Cook. A survey of methods for time series change point detection. Knowledge and Information Systems, 51 (2017), 339-367.

[2] S.-W. Cheng, O. Cheong, and T. Lee. Fitting a graph to one-dimensional data. Proceedings of the 32nd Canadian Conference on Computational Geometry, 2020.

[3] S.I. Daitch, J.A. Kelner, and D.A. Spielman. Fitting a graph to vector data. Proceedings of the 26th International conference on Machine Learing, 2009, 201-208.

[4] S. Han, H. Huang, H. Qin, and D. Yu. Locality-preserving L1-graph and its application in clustering. Proceedings of the 30th Annual ACM Symposium on Applied Computing, 2015, 813-818.

[5] S. Hido, T. Idé, H. Kahsima, H. Kubo, and H. Matsuzawa. Unsupervised change analysis using supervised learning. Proceedings of the Pacific-Asia Conference on Knowledge Discovery and Data Mining, 2008, 148-159.

[6] T. Jebara, J. Wang, and S.-F. Chang. Graph construction and b-matching for semi-supervised learning. Proceedings of the 26th International conference on Machine Learing, 2009, 441-448.

[7] R. Klinkenberg and T. Joachims. Detecting concept drift with support vector machines. Proceedings of the 17th International Conference on Machine Learning, 2000, 487-494.

[8] W.-H. Lee, J. Ortiz, B. Ko, and R. Lee. Time series segmentation through automatic feature learning. arXiv:1801.05394v2, 2018.

[9] W. Liu, J. He, and S.-F. Chang. Large graph construction for scalable semi-supervised learning. Proceedings of the 27th International Conference on Machine Learning, 2010, 679-686.

[10] R.D.C. Monteiro and I. Adler. Interior path following primal-dual algorithms. Part II: convex quadratic programming. Mathematical Programming, 44:43-66, 1989.

[11] L. de Moura and N. Bjørner. Z3: an efficient SMT solver. Proceedings of the 14th International Conference on Tools and Algorithms for the Construction and Analysis of Systems, 2008, 337-340. Codes available at https://github.com/Z3Prover/z3.

[12] A.Y. Ng, M.I. Jordan, and Y. Weiss. On spectral clustering: analysis and an algorithm. Proceedings of the 14th International Conference on Neural Information Processing Systems, 2001, 849-856.

[13] M. Scholz and R. Klinkenberg. Boosting classfiers for drifting concepts. Intelligent Data Analysis Knowledge Discovery from Data Streams, 11 (2007), 3-28. 
[14] S. Xiang, F. Nie, and C. Zhang. Semi-supervised classification via local spline regression. IEEE Transactions on Pattern Analysis and Machine Intelligence, 32 (2010), 2039-2053.

[15] Y.-M. Zhang, K. Huang, and C.-L. Liu. Learning locality preserving graph from data. IEEE Transactions on Cybernetics, 44 (2014), 2088-2098.

[16] D. Zhou, O. Bousquet, T.N. Lal, J. Weston, and B. Schölkopf. Learning with local and global consistency. Proceedings of the 16th International Conference on Neural Information Processing Systems, 2003, 321-328. 